
allemande

45-2 | 2013

Images et discours de la nation

\title{
Religion als Gegenkultur in der DDR?
}

Eine Untersuchung des Umgangs mit Kirchenbau in der DDR am Beispiel Eisenhüttenstadt

Sylvie Le Grand

\section{(2) OpenEdition}

1 Journals

Édition électronique

URL : https://journals.openedition.org/allemagne/1770

DOI : 10.4000/allemagne. 1770

ISSN : 2605-7913

Éditeur

Société d'études allemandes

\section{Édition imprimée}

Date de publication : 30 décembre 2013

Pagination : 457-470

ISSN : 0035-0974

\section{Référence électronique}

Sylvie Le Grand, "Religion als Gegenkultur in der DDR?", Revue d'Allemagne et des pays de langue allemande [Online], 45-2 | 2013, Online erschienen am: 29 Juli 2019, abgerufen am 19 Mai 2021. URL: http://journals.openedition.org/allemagne/1770 ; DOI: https://doi.org/10.4000/allemagne.1770 


\section{Religion als Gegenkultur in der DDR? Eine Untersuchung des Umgangs mit Kirchenbau in der DDR am Beispiel Eisenhüttenstadt}

\section{- Sylvie Le Grand*}

Im Jahre 1983 erschien in der DDR ein 78seitiges, anschauliches und gut illustriertes Büchlein in französischer Sprache: Les chrétiens et les Églises, information sur la République démocratique allemande. Es ist eine klar gegliederte und solide informierte, offizielle Broschüre der Auslandsagentur Panorama DDR. Ziel war es, dem Ausland zu zeigen, wie harmonisch die Beziehungen zwischen Marxisten und Christen und wie gut integriert die Christen und ihre Kultur in der DDR-Gesellschaft waren ${ }^{(1)}$. Dass ausgerechnet Eisenhüttenstadt (unter der Rubrik „materielle Lage“ [situation matérielle] zum Thema Wiederaufbau bzw. Neubau von Kirchen) erwähnt wurde, ist nicht ohne unwillkürliche Ironie, war doch die Entstehungsgeschichte dieser Gemeinde im Hinblick auf ihren Kirchenbau eine recht langwierige, wie wir im Folgenden zeigen wollen.

Doch ist eine solche Propaganda zunächst einmal vor dem Hintergrund des kirchlich und staatlich mit viel Pomp gefeierten Luther-Jahres und darüber hinaus der veränderten politischen und gesellschaftlichen Rahmenbedingungen der Staat-Kirche-Beziehungen zu verstehen.

Wie verhielt sich aber tatsächlich der DDR-Staat im Laufe seines 40jährigen Bestands zur Frage des christlichen Erbes und im Umgang mit den verschiedenartigen christlichen Kulturgütern, die im Osten Deutschlands als historischer Wiege des deutschen Protestantismus so zahlreich waren? Dies ist eine der Fragen, die mich bei dieser

* Maître de conférences à l'Université Paris Ouest Nanterre La Défense.

1 Unter der Rubrik „materielle Lage“ (situation matérielle) wird unter anderem auf die vielfältigen Bemühungen des Wiederaufbaus und Neubaus von kirchlichen Gebäuden, wie z.B. in Eisenhüttenstadt hingewiesen, sowie auf die große Rolle, die kulturelles Erbe (patrimoine culturel) und zeitgenössische Kunst (création contemporaine) im geistig-religiösen (vie spirituelle) Leben spielen. En exclusivité. Les chrétiens et les Églises. Information sur la République démocratique allemande, Panorama DDR, Auslandspresseagentur GmbH Verlag Zeit im Bild, $1^{\text {er }}$ mars 1983, 79 Seiten + Ill. (hier S. 65-66). 
Untersuchung ${ }^{(2)}$ geleitet haben. Auf diesem Umweg möchte ich hier die Relevanz des gegenkulturellen Ansatzes in Bezug auf die Kirchen in der DDR überprüfen.

Indes habe ich auf einen ziemlich klassischen Kulturbegriff zurückgegriffen, d.h. zunächst unter Kultur die historisch überlieferten Kunstprodukte und Bauwerke, Bildungserscheinungen, Lebensweisen, Vorstellungen, die von bestimmten Milieus (hier kirchlichen Milieus) getragen bzw. verkörpert waren und eventuell weiter tradiert wurden, ohne jedoch den normativen Gehalt zu übernehmen, der diesem Kulturbegriff als „vom Bürgertum reklamiert“ im 19. Jahrhundert anhaftete ${ }^{(3)}$. Somit habe ich die Religion als möglichen Bestandteil einer Kultur und daher im weitesten Sinne verstanden: als soziale Institution, als System von Glaubensinhalten, Überzeugungen und Riten, sowie als Bewusstseinselement.

Eine solche Perspektive über Kultur- und Religionsbegriffe bot sich hier aus verschiedenen Gründen an:

- Gerade auf der Grundlage dieses Kulturbegriffs ist im weiten Feld der Beziehungen und Wechselwirkungen zwischen Religion und Kultur bzw. Protestantismus und Kultur die starke Strömung des Kulturprotestantismus im 19. Jahrhundert entstanden, als Bildungsreligion, die die Grenzen zwischen Religion und Kultur weitgehend verwischt. Auf dieses uferlose Aufgehen der Transzendenz in der Immanenz hat Karl Barth im 20. Jahrhundert mit seiner dialektischen Theologie scharf reagiert ${ }^{(4)}$. Diese - hier stark schematisierten - Pole bilden zwei extrem entgegengesetzte Positionen im Protestantismus, in deren Spannungsfeld sich das Verhältnis zwischen Religion und Kultur ausbildet.

- Nach Gründung der DDR wurde spätestens mit dem Aufbau des Sozialismus ab Juli 1952 dem Bürgertum und seiner als dekadent verpönten Kultur der Kampf angesagt. Auf der Tagesordnung stand nun die Ausbildung des sozialistischen Menschen, in dem die religiösen sprich christlichen Bindungen nichts zu suchen hatten und demnach systematisch bekämpft wurden. Ihr Absterben wurde vorprogrammiert. Die Religion und ihre kulturellen Erscheinungen wurden faktisch als eine Art Gegenkultur im

2 Dieser Aufsatz beruht weitgehend auf dem Vortrag, den ich am 18.5.2011 an der Universität Toulouse Le Mirail im Rahmen der von Françoise Knopper und Mechthild Coustillac organisierten Tagung „Contre-cultures en RDA?/ Gegenkulturen in der DDR?“ gehalten habe. Den Organisatorinnen der Tagung möchte ich hier für ihren Impuls, mich mit diesem Ansatz zu beschäftigen, danken.

3 Ansgar NüNning (Hg.), Grundbegriffe der Kulturtheorie und Kulturwissenschaften, Stuttgart, Metzler, 2005, S. 112.

4 In einem kurzen Gedenkartikel der EKD vom 3.5.2011 „Evangelischer Kirchenvater des 20. Jahrhunderts. Vor 125 Jahren wurde Karl Barth geboren“ wird daran erinnert, dass Karl Barth „ab den 1920er Jahren zusammen mit Friedrich Gogarten, Rudolf Bultmann, Emil Brunner und anderen die ,Dialektische Theologie [entwickelte], bei der die für ihn unüberwindbaren Gegensätze Gott und Mensch oder Zeit und Ewigkeit im Mittelpunkt standen. Barth brach radikal mit einer Theologie, die zwischen Gott und Mensch vermitteln oder den - aus seiner Sicht unendlichen - Abstand zwischen Gott und Mensch verringern wollte. [...] Barth [...] spricht von Gott als dem ,ganz Anderen“', http://www.ekd.de/print. php?file=aktuell/76401.html (abgerufen am 9.5.2011). Grundlegend dazu Heinz Zahrnt, Die Sache mit Gott. Die protestantische Theologie im 20. Jahrhundert, München, Piper, 1966 (Taschenbuchneuausgabe 1988), 427 S. Siehe u.a. „die Umkehrung Schleiermachers“, S. 38ff: „Karl Barths ,Römerbrief“ ist von der ersten bis zur letzten Seite ein einziger großer Protest gegen die theologische Ära, die vor allem durch die Namen Schleiermacher, Ritschl, Harnack und Troeltsch bestimmt war.“ 
wortwörtlichen Sinne betrachtet, die es auszutilgen galt ${ }^{(5)}$. Hatte ja nicht Bebel gesagt, dass Sozialismus und Christentum sich wie Feuer und Wasser verhalten? So wurden im vulgären Marxismus bzw. Marxismus-Leninismus und im wissenschaftlichen Atheismus diese beiden Größen als Gegenpole, Gegenwelten, ihrem jeweiligen absoluten Wahrheitsanspruch entsprechend hochstilisiert, der aus beiden konkurrierende Heilslehren machte.

Dieser Aspekt der DDR-Ideologie und Kirchenpolitik rechtfertigt einen zeitweiligen Gebrauch - gleichsam als Arbeitsbegriff - von Gegenkultur in dem eben skizzierten wörtlichen Sinne: wir stehen in der DDR einem gesellschaftlichen Versuch gegenüber, wonach eine Umkehrung der Verhältnisse zwischen Leit- und Randkultur unternommen wurde. Doch ist es fraglich, ob dieses binäre Denken durchgehend auf jede Periode, jedes Fallbeispiel, jede Ebene der Kirchenpolitik und umgekehrt auf das Selbstverständnis der Kirchen bezogen werden kann. Die SED konnte schließlich nicht gänzlich auf Tradition und auf das kulturelle Potential des Christentums verzichten.

Wir möchten diesen Begriff folglich hier auf seine geschichtliche Relevanz hin überprüfen und untersuchen, ob und inwiefern er kompatibel ist mit der Zwischenstellung der Kirchen in der DDR ${ }^{(6)}$.

Das Thema Gegenkultur möchte ich also nicht unter dem vielleicht naheliegenderen, weil viel bearbeiteten Aspekt des Schutzes der oppositionellen Gruppen bzw. der subkulturellen Szene durch die Kirchen (als Dach für Gegenkultur im angelsächsischen Sinn von counter culture) untersuchen, sondern am Beispiel des Umgangs der DDR mit den kirchlichen Kulturgütern, d.h. hier in erster Linie am Beispiel Kirchenbau, Kirchenabriss, Kirchenrenovierung, Denkmalpflege behandeln.

Warum gerade die Kirchenbaufrage?

Als „,architektonischer und damit materialisierter Ausdruck der religiösen Idee “ ${ }^{(7)}$ kristallisiert Kirchenbau städtebauliche, ästhetische, theologische und nicht zuletzt politische Gesichtspunkte, zumal er als materielle Kulturerscheinung öffentlichen Charakter hat, was einen Teil seiner Brisanz in der DDR ausmachte. Der Kirchenbau hinterlässt zudem Spuren aus der Vergangenheit, vereinigt Gegenwart und Vergangenheit, entspricht auch dem Gemeinschaftsbild der Kirche oder der Gemeinde als Körper bzw. als Baugefüge mit verschiedenen Elementen. Kurz, er vereinigt Kult und Kultur!

Zunächst gehen wir vom Fallbeispiel Eisenhüttenstadt ${ }^{(8)}$ aus, um dann in einem zweiten Schritt diese einmalige Geschichte vor dem Hintergrund anderer Entwick-

5 In der üblichen DDR-Terminologie hie $\beta$ es staatsfeindlich oder gegenrevolutionär.

6 Diese Zwischenstellung ist auf mindestens drei Faktoren zurückzuführen: auf die politische Weichenstellung durch die Sowjets, die die Kirchen relativ wohlwollend behandelten, ihnen die Bodenreform ersparten und günstige verfassungsrechtliche Bedingungen ähnlich wie in der BRD und in Anlehnung an die Weimarer Republik schufen; auf die deutsch-deutsche Situation im doppelten Deutschland; auf allerlei pragmatische Erwägungen, die die konkrete Kirchenpolitik jenseits der atheistischen Prinzipien prägten.

7 Elisabeth Knauer-Romani, „Kirchenbau in Stalinstadt“, in: Holger Barth (Hg.), Projekt Sozialistische Stadt: Beiträge zur Bau- und Planungsgeschichte der DDR, Berlin, Reimer, 1998, S. 211-217 (Zitat S. 211).

8 Ausgangspunkt dieser Untersuchung sind die Erinnerungen von Pfarrer Heinz Bräuer gewesen: Die ersten drei Jahrzehnte der evangelischen Friedenskirchengemeinde Eisenhüttenstadt. Erinnerungen, Eisenhüttenstadt, 1991, 543 S. (im Internet nun als freie Datei zugänglich). Von dieser Quelle ausgehend habe ich 
lungslinien (Kirchenbau allgemein, Staat-Kirche-Beziehungen und Kirchenpolitik allgemein, politische Großwetterlage) zu betrachten und in größere Zusammenhänge einzubetten.

\section{Eisenhüttenstadt, Stadt und Kombinat - ein paar Daten ${ }^{(9)}$}

„In der Wohnstadt des EKO geboren, in Stalinstadt getauft, und in Eisenhüttenstadt zur Kirchenweihe gegangen, ohne einmal umgezogen zu sein“: dieses Bonmot von Pf. Bräuer beleuchtet den dreifachen Namenwechsel der neuen Stadt und lässt darüber hinaus ihre wechselvolle Geschichte ahnen.

Als erste sozialistische Stadt in der DDR eignet sich Eisenhüttenstadt ganz besonders für eine Untersuchung der DDR-Verhältnisse, da einiges dort wie in einem Brennglas erscheint. Die neue Stadt war ja Vorzeigestadt des Regimes, wo der Mensch und die Familie neuen Typus erprobt werden sollten, eine Art Aushängeschild für den Aufbau des Sozialismus in der DDR.

Die neue Stadt entstand aufgrund eines Parteibeschlusses von 1950 und war als Wohnstadt des neuen Eisenhüttenkombinats konzipiert. Nach dem Stahlembargo durch die Westmächte 1950 brauchte die DDR eine metallurgische Basis zur Stahlerzeugung. Aufgrund der sowjetischen Doktrin der wirtschaftlichen Selbstversorgung der Satellitenstaaten wurden Beschlüsse zur Schaffung eines neuen Industriekomplexes gefasst.

Das neue Werk zog junge Arbeiter aus der ganzen DDR an und wurde zum Schmelztiegel der neuen sozialistischen Gesellschaft. In den 1950er Jahren lag das Durchschnittsalter der Einwohner bei 25 Jahren; durch den Umzug waren diese Arbeiter ohne familiäre und traditionelle Bindung, was für die Formung des neuen sozialistischen Menschen günstige Voraussetzungen schuf. Die großzügig und modern angelegten Wohnungen in der neuen Stadt waren sehr begehrt, zumal die allgemeine Wohnlage in der DDR lange Zeit - und insbesondere für junge Leute - angespannt war.

Geplant für 30.000 Menschen zählte die Wohnstadt 1955 schon 15.157 Einwohner und bis 1960 sogar 24.372 Menschen. Sie lag unweit der Oder zwischen der Kleinstadt

im Evangelischen Zentralarchiv (EZA) und im Evangelischen Landeskirchlichen Archiv Berlin (ELAB) Unterlagen zur Kirchengemeinde in Eisenhüttenstadt und zum Kirchenbau stichprobenartig konsultiert, sowie vor Ort Gespräche geführt mit den langjährigen Mitgliedern des Gemeindekirchenrats, Klaus und Christel Jachning, dem Haus- und Kirchwart Hans-Peter Mikloweit. In einer breiteren Perspektive, der ich in den Grenzen des vorliegenden Beitrags kaum Rechnung tragen kann, aber einen anderen Aufsatz widme „Le patrimoine culturel chrétien en RDA: un potentiel critique?“, in: Hélène CAMARADE, Sibylle Goepper (Hg.), Actes du colloque „Résistance et opposition en RDA: tentatives de conquête de l'espace public (1949-1990)“ (à paraître), habe ich mich auch mit wichtigen Akteuren der staatlichen Denkmalpflege, Herrn Dr. Peter Goralczyk (u.a. 1987-90 Generalkonservator der DDR) und Herrn Prof. Dr. Ernst Badstübner (Kunsthistoriker), und einem Vertreter des berlin-brandenburgischen kirchlichen Bauamts, Herrn Dipl.-Architekt Heinz Tellbach unterhalten. Ihnen sei hier für ihre Gesprächsbereitschaft und Hilfe herzlich gedankt. Dem Andenken meines Freundes, Kirchenmusiker Wolfgang Fischer (1932-2011), mit dem ich zusammen mit seiner Frau Dorrit immer wieder sehr anregende Diskussionen zum (kirchlichen) Leben in der DDR führen durfte, möchte ich diesen Beitrag widmen.

9 Ein großer Teil dieser Daten sind den Erinnerungen von Pf. Heinz Bräuer und der Arbeit von Michael Tillmann entnommen. Michael Tillmann, Das Verhältnis von Staat und Kirche am Beispiel Stalinstadt in den fünfziger Jahren, Schriftliche Hausarbeit im Rahmen der Ersten Staatsprüfung für das Lehramt Geschichte/Deutsch Sekundarstufe II/I (Themensteller: Christoph Kleßmann), Universität Potsdam, Februar 1995, 105 S. (Typoskript, über die Fernleihe der Universität Tübingen zugänglich). 
Fürstenberg und dem Dorf Schönflie $\beta$, wo im Gegensatz zur neuen Stadt noch traditionelle Milieus und zu dieser Zeit auch Privatgeschäfte oder Privathandwerker zu finden waren. Dass die neue Stadt Vorbildcharakter haben sollte, zeigt der Umstand, dass ursprünglich Kleingartenkolonien als Relikt der bürgerlichen Zeit ausgeschlossen waren: „es lie $\beta$ sich auf die Dauer ebenso wenig durchhalten wie der Bann über die Kirchen“"(10), Kleingärten wurden 1959 zugelassen.

Dass das Primat des Politischen dort besonders galt, kam unter anderem in der ideologisch überhöhten Bezeichnung des Kombinats und seiner Produktion als Friedenswerk, Friedensstahl, Friedenstadt zum Ausdruck! Nicht ohne Ironie ist dann 1963 die Auswahl eines Namens für die Gemeinde durch den Gemeindekirchenrat auf Friedensgemeinde gefallen. Die Aufbruchsstimmung und Euphorie der 1950er Jahre fand außerdem ihren Niederschlag in Gedichten und Liedern (u.a. Hans Marchwitza, „Roheisen“, Oskar Nerlinger, „Mit Pinsel und Feder im Eisenhüttenkombinat Ost“), Festen (den seit 1954 jährlich inszenierten Hüttenfesten), einem Bildband (Neues Leben, Neue Menschen, 1958).

\section{Rückblick auf die langwierige Entstehungsgeschichte eines evangelischen Massivkirchenbaus in Eisenhüttenstadt}

Dass kirchliches Leben in Stalinstadt/Eisenhüttenstadt unerwünscht war, lässt sich in erster Linie an der langwierigen Geschichte des Kirchbaus ablesen. Es ist die Geschichte einer 30jährigen Verzögerung, eines dauerhaften Provisoriums und eines ständigen Vertröstens auf eine ungewisse Zukunft.

Als der erste Pfarrer der noch nicht existierenden Gemeinde am 1.2.1953 berufen wurde, lag seit Herbst 1952 ein Wagen der Goßner Mission auf einem gepachteten Privatgrundstück und diente als Raum für Gottesdienst und jedwedes Treffen für die im Entstehen begriffene Gemeinde. In der Zeit der schärfsten Auseinandersetzungen um Junge Gemeinde und Kirchenarbeit in der DDR wurde dieser Wagen zwei Monate lang vom Staat beschlagnahmt. Ein 150 Menschen fassendes Zelt wurde von der Kirchgemeinde zeitweilig beigefügt. Obwohl in den anfänglichen Bebauungsplänen des Generalprojektanten Kurt W. Leucht ${ }^{(11)} 1952$ und 1953 noch genaue Standorte für eine evangelische und eine katholische Kirche gezeichnet waren und sogar großzügig angelegte Pläne vorlagen, leugnete der Architekt später, 1957 und 1960, eine solche Planung je entworfen zu haben.

Verschiedene Versprechungen wurden jeweils gegeben im Hinblick auf einen Massivbau $^{(12)}$, auf die Bewilligung eines Standorts, auf das zur Verfügung Stellen eines

10 Rosmarie Beier (Hg.), Aufbau West, Aufbau Ost. Die Planstädte Wolfsburg und Eisenhüttenstadt in der Nachkriegszeit (Buch zur Ausstellung des Deutschen Historischen Museums vom 16. Mai bis 12. August 1997), Ostfildern-Ruit, G. Hatje, 1997, S. 255-256.

11 Zur wechselvollen Biographie von Kurt W. Leucht (1913-2001) siehe die interessanten Ausführungen von Jörn Schütrumpf, „Kurt W. Leucht, Planer von Stalinstadt, der ,ersten sozialistischen Stadt Deutschlands“, in: R. BeIER (Hg.), ebd., http://www.dhm.de/ausstellungen/aufbau_west_ost/katlg09.htm (abgerufen am 8.7.2013).

12 „Massivbau“ ist der Terminus, der von den Betroffenen benutzt wurde, um den Unterschied zum langwährenden Provisorium der Baracke (nach dem Wohnwagen und Zelt!) zu markieren. 
Grundstücks (sei es vom Staat gepachtet oder im Rahmen eines Tausches verkauft), dann aber mehrfach nicht eingehalten, so dass der Eindruck einer undurchschaubaren Situation mit vielen Ungereimtheiten und Widersprüchen zwischen den verschiedenen beteiligten Ebenen entsteht. Während das Ministerium für Aufbau und die städtische Bauleitung der Kirche eher wohlgesonnen zu sein schienen, wurde in der Partei auf Stadt- oder Bezirksebene oft eine härtere Linie vorgegeben ${ }^{(13)}$.

Dieses Wirrwarr und Durcheinander hielt auch nach dem Besuch von Walter Ulbricht am 7.5.1953 zur Namensnennung der neuen Stadt und seiner sogenannten „Turmrede“ an: darin erteilte er unmissverständlich jedem Kirchbau eine Absage, indem er den „kapitalistischen Verdummungseinrichtungen“ in der Stalinstadt jede Existenz absprach. „Andere Türme [als fürs Rathaus oder Kulturhaus] können wir in der sozialistischen Stadt nicht gebrauchen“(14).

Die Kirche suchte und bekam die Unterstützung vom damaligen Vorsitzenden der CDU (Ost), dem stellvertretenden Ministerpräsidenten bzw. stellvertretenden Vorsitzenden des Ministerrats, Otto Nuschke, der auch bis 1957 für die damalige Hauptabteilung „Verbindung zu den Kirchen“(15) verantwortlich war, sowie von Propst Grüber, der von 1949 bis Mai 1958 EKD $^{(16)}$-Bevollmächtigter bei der DDR-Regierung war.

Aufgrund von ersten positiven Versprechungen wurde von der Kirche eine sogenannte Kirchbaracke gekauft, aber es dauerte wieder eine Weile, bis der Standort gefunden und die Baugenehmigung erteilt wurde. Der Durchbruch kam 1954, als die Kirch- und Pfarrbaracken aus Holz aufgestellt werden konnten. Sie blieben dann bis zur Grundsteinlegung des Gemeindezentrums 1976 erhalten. Dieses Gemeindezentrum ist ein Produkt des Sonderbauprogramms, das im Kontext der veränderten Rahmenbe-

13 Diese leichten Kursunterschiede mögen zeitliche Gründe gehabt haben: manchmal dauerte es eine Weile bis eine Parteientscheidung den städtischen Ausführenden erreicht hatte, daher die verwirrende Situation.

14 M. Tillmann, Das Verhältnis von Staat und Kirche (Anm. 9), S. 19, Fußnote 59: Hinweis auf Kirchliches Jahrbuch 1953, S. 184.

15 Diese Verwaltungsstelle, die von 1949 bis zum Tod von Otto Nuschke am 27.12.1957 bestand, war der SED ein Dorn im Auge. Auch den Sowjets erschien sie als eine Agentur der Kirchen im Staatsapparat. Nach ihrer Auflösung nach Nuschkes Tod bestand die staatliche Kirchenpolitik vornehmlich in der Aktivität der im März 1957 neu gegründeten Dienststelle des Staatssekretärs für Kirchenfragen. Laut Martin Goerner machte Otto Nuschke dem Namen seines Amtes Ehre, indem er tatsächlich in Verbindung zu den Kirchen stand. Er unterhielt komplexe Beziehungen zur CDU. Von der SED wurde er persönlich als wichtiges deutschlandpolitisches Bindeglied relativ verschont. Martin Georg Goerner, „Die Behandlung der Kirchenpolitik im Staatsapparat und in den Massenorganisationen“, in: Clemens Vollnhals, Die Kirchenpolitik von SED und Staatssicherheit. Eine Zwischenbilanz, Berlin, Links, 1997 (2. durchgesehene Auflage), S. 139-158; Siehe dazu auch Andreas ScHALÜcK, Eine Agentur der Kirchen im Staatsapparat? Otto Nuschke und die Hauptabteilung „Verbindung zu den Kirchen“ 1949-1953, Berlin, Akademie Verlag, 1999, 343 S.

16 Die Evangelische Kirche in Deutschland (EKD) entstand 1945 in Treysa als Zusammenschluss der evangelischen Landeskirchen in Ost- und Westdeutschland und gab sich im Juli 1948 in Eisenach eine Grundordnung. Erst im Juni 1969 trennten sich organisatorisch die evangelischen Landeskirchen in Ost und West und es entstand neben der EKD der Bund der evangelischen Kirchen in der DDR (BEK) als Zusammenschluss der 8 evangelischen Landeskirchen in der DDR. Die Grundordnungen von EKD und BEK verwiesen gegenseitig auf die „besondere Gemeinschaft“ mit der jeweiligen anderen Dachorganisation. Der BEK löste sich 1991 auf. 
dingungen für Staat-Kirche-Beziehungen Anfang der 1970er Jahre ausgehandelt und beschlossen wurde. Wir kommen gleich darauf zurück.

In der Zwischenzeit hatte sich im Zuge der späten Entstalinisierung ab 1961 die Lage etwas entspannt ${ }^{(17)}$ : durch die Zusammenlegung der Städte Fürstenberg und Stalinstadt mit Schönflie $\beta$ hatte sich die Frontlinie etwas verlagert, es ging nun um den (Massiv) Bau einer zweiten evangelischen Kirche in Eisenhüttenstadt, da doch die im Krieg stark beschädigte Kirche von Fürstenberg noch vorhanden war und renoviert werden sollte.

Bestimmt durch die patriarchalische Figur von Pfarrer Bräuer war das Leben der Gemeinde eher traditionell ausgerichtet; nichts war dort vom sogenannten „Teamdenken“ und "Gemeindeaufbaukonzept“ zu spüren, das vom Bund der evangelischen Kirchen in der DDR (BEK) gefördert und in etlichen anderen evangelischen Neubaugemeinden der DDR umgesetzt wurde ${ }^{(18)}$. Hatte das mit der Ausnahmesituation von Eisenhüttenstadt zu tun? Mit der Persönlichkeit des Pfarrers? Fest steht, dass auch auf Parteiebene $^{(19)}$ die Stadt zwischen Mitte der 1950er und Anfang der 1960er Jahre aus der Kirchenpolitik des Bezirks herausgehalten wurde ${ }^{(20)}$.

Das 1981 eingeweihte Gemeindezentrum war im Anschluss an die architektonische Entwicklung im Kirchenbau als Mehrzweckgebäude konzipiert. Nicht nur aus politischen Gründen, sondern auch im Zusammenhang mit dem damals in Ost und West feststellbaren Trend zur multifunktionalen Bauweise wurde auf Kirchturm und auf die Bezeichnung als Kirche verzichtet. Die Grundstücksfrage (Tausch zwischen Staat und Kirche; Eintragung des Kircheneigentums ins Grundbuch) wurde erst 1985 geklärt.

Der Eindruck, den man angesichts dieser abenteuerlichen Baugeschichte gewinnt, ist eher der von Schikanen und Willkür, aber nicht von massiven Repressalien. Die katholische Gemeinde hat es anscheinend schwerer gehabt, ihre Baracke wurde in einer nächtlichen Aktion im März 1954 zerstört $^{(21)}$, ein Massivbau ist in der DDR-Zeit nie entstanden.

17 Laut Michael Tillmann (Das Verhältnis von Staat und Kirche [Anm. 9], S. 5) wurden die Auseinandersetzungen weniger scharf und der sozialistische Charakter der Stadt verblasste.

18 Siehe dazu meine Untersuchung zum Vergleich zweier Neubaugemeinden in Halle-Neustadt und Dortmund-Scharnhorst: Sylvie LE GRAND, „Kirchenalltag in Ost und West: die Gemeinden“, in: Claudia LEPP, Kurt Nowak (Hg.), Evangelische Kirche im geteilten Deutschland (1945-1989/90), Göttingen, Vandenhoeck \& Ruprecht, 2001, S. 228-254.

19 Zum Beispiel wurde der Pfarrer nach der Unterredung zwischen Innenminister Maron und Kirchenvertretern am 10.2.1956 von den Gesprächen auf Bezirksebene (Frankfurt/Oder) ausgenommen, die mit allen Pfarrern durchgeführt werden sollten. M. Tillmann, Das Verhältnis von Staat und Kirche (Anm. 9), S. 6 (Fußnote 13); S. 20.

20 Auf einer anderen Ebene und unter veränderten Rahmenbedingungen ist festzustellen, dass diese Kirchgemeinde, vielleicht bedingt durch die politisch exponierte Lage der Stadt ein zwar lebendiges, aber am Rande vielfacher innerkirchlicher Entwicklungen bestehendes Leben führte, so dass jedenfalls Erscheinungen der späten 1970er und 1980er Jahre an ihr vorbei zu verlaufen schienen, wie z.B. der Konflikt um den kirchlichen Aufnäher Schwerter zu Pflugscharen Anfang der 1980er oder das Gruppenphänomen Mitte bis Ende der 1980er Jahre. Im Kirchenkreis Guben, dem die Stadt Forst sowie Eisenhüttenstadt angehörten, wurden zwar die Konflikte über die Forster Aktivitäten um das Samisdatblatt Aufbruch o.ä. diskutiert, aber ohne direkte Auswirkung auf das Gemeindeleben in Eisenhüttenstadt.

21 H. BRÄUER (Pfarrer a.D.), Erinnerungen (Anm. 8), S. 89-90 (mit Bildern der zerstörten Baracke). 


\section{Einbettung in größere Zusammenhänge - Gegenüberstellung von verschiedenen Entwicklungslinien}

Vor welchem Hintergrund ist das Zustandekommen des Gemeindezentrums in Eisenhüttenstadt zu verstehen? Zunächst ist der Zusammenhang mit der Auflockerung des Staat-Kirche-Verhältnisses wichtig: nach dem Mauerbau und der damit erreichten relativen inneren Stabilisierung der DDR ließen sich Anzeichen einer Annäherung an christliche Kräfte von Seiten der SED erkennen: Ulbricht sprach in einer Rede vor der Volkskammer im Oktober 1960 von der humanistischen Verantwortung von Marxisten und Christen; eine weitere Stufe eines zur Schau gestellten Integrationswillens von Christen wurde vom Politbüro-Mitglied Albert Norden zwölf Jahre später erreicht, als er in einem Grußwort des ZK der SED an den 13. Parteitag der CDU (Ost) in Dresden den Begriff vom sozialistischen Staatsbürger christlichen Glaubens prägte ${ }^{(22)}$.

Diese Auflockerung des Gegensatzes zwischen Staat/Partei und Kirche hatte aber nur taktischen Charakter: sie gehörte zur Differenzierungsstrategie der SED, die durch Entgegenkommen Unterstützung oder mindestens Loyalität in den Reihen der Kirchen zu gewinnen versuchte. Solche scheinbare Annäherungsversuche konnte sich die SED nun erlauben, nachdem sie ihren Feind teilweise erledigt zu haben glaubte, d.h. nachdem ihre Entkirchlichungsversuche in den eigenen Reihen und in der Bevölkerung im Zuge des Konfliktes um die Junge Gemeinde (1953) und die Einführung der Jugendweihe (1954) einigermaßen erfolgreich gewesen waren.

Nachdem die Trennung von der EKD erreicht und eine DDR-spezifische kirchliche Vertretung des Protestantismus, der BEK im Juni 1969 gegründet worden war, wurde diese Strategie um so stärker fortgesetzt; sie nahm sogar noch unbekannte Ausmaße an: es hatte nun - auf beiden Seiten übrigens - die „Stunde der Pragmatiker“ geschlagen ${ }^{(23)}$.

Das sogenannte Sonderbauprogramm ist ein gutes Beispiel dafür. Es ist ein noch nicht richtig erforschtes Gebiet der kirchlichen Zeitgeschichte. Schon 1970 und 1971 gab es erste Gespräche vom Sekretär des BEK, Manfred Stolpe, mit „Freunden aus der EKD“, zur Besprechung einer entscheidenden Hilfe der Schwesterkirchen im Westen für ein Kirchensonderbauprogramm in der DDR. Die jährlichen Bauzuschüsse des Staates in Höhe von zirka 800.000 Mark, die für denkmalpflegerische Zwecke gegeben wurden, reichten nicht aus angesichts der großen Baulast im Osten (über 4000 Gemeinden mit einer Kirche fast überall), so Stolpe rückblickend $1976^{(24)}$. Der BEK überreichte Listen von Kirchen, die jeweils dem Staat abzugeben bzw. zur Verfügung zu stellen, zu renovieren oder gar aus dem Boden zu stampfen waren. Durch Beschluss des Ministerrates der DDR vom 13.12.1972 wurde dann die Durchführung eines Renovierungs- oder Bauprogramms für die Jahre 1973-75 für 45 Kirchen genehmigt. Es wurde mehrfach

22 Frédéric Hartweg (Hg.), SED und Kirche. Eine Dokumentation ihrer Beziehungen, Bd. 2: 1968-1989, bearbeitet von Horst Dohle, Neukirchen-Vluyn, Neukirchener, 1995, S. 620.

23 Dieser Ausdruck wurde auf die Phase nach dem Spitzengespräch zwischen Staat und Kirche am 6.3.1978 nachträglich gemünzt. Joachim HeISE, „Kirchenpolitik von SED und Staat zwischen ideologischem Dogma, praktischer Toleranz und politischem Mißtrauen“, in: Horst DÄHN (Hg.), Die Rolle der Kirchen in der DDR- Eine erste Bilanz, München, Olzog, 1993, S. 79.

24 Bund der Evangelischen Kirchen in der DDR, Sonderbauprogramm. Zwischenbericht, im Mai 1976, S. 1. Diese Dokumentation ist im Evangelischen Zentralarchiv Berlin zu konsultieren. 
verlängert: 1976-80, dann bis 1985 und zuletzt bis 1992, so der Bericht $1988^{(25)}$. Mit Ausnahme eines einzigen Falls, des Gemeindezentrums von Stralsund Knieper West in der Landeskirche von Greifswald ${ }^{(26)}$ wurden Gemeindezentren für Neubaugebiete erst ab 1976 in Aussicht genommen ${ }^{(27)}$.

Das Programm hing von der großzügigen Spendenbereitschaft der EKD ab, der sich auch Schweden und die schweizerische Eidgenossenschaft punktuell anschlossen ${ }^{(28)}$. Im Grunde aber flossen in erster Linie Gelder vom Bundesministerium für innerdeutsche Beziehungen. Unterhändler auf westlicher Seite war u.a. der Vizepräsident des Diakonischen Werkes, Ludwig Geißel, auf östlicher Seite Manfred Stolpe.

Der Kern der ganzen Angelegenheit beruhte für den DDR-Staat auf dem wachsenden Bedürfnis nach Devisenbeschaffung. Zuständig dafür war die 1966 gegründete Abteilung KoKo (Kommerzielle Kontakte, bzw. kommerzielle Koordinierung) im Ministerium für Außenhandel ${ }^{(29)}$. Sie war von Alexander Schalck-Golodkowski geleitet und ein Teil ihrer Tätigkeit war auf die Arbeit mit den Kirchen ausgerichtet ${ }^{(30)}$. Als das Sonderbauprogramm ausgehandelt wurde, war die DDR an einer Ausweitung der Transferleistungen interessiert, und die Kirche an einem Bauleistungsfonds. Laut Zeugnis von Manfred Stolpe erfolgte der entscheidende Durchbruch im Zusammenhang mit der Berliner Domfrage. Im Zuge der Fertigstellung des Berliner Stadtzentrums und des 1972 geplanten Baus des Palastes der Republik war der Staat plötzlich an der schnellen Renovierung des Berliner Doms interessiert, was hingegen die eigentlichen Eigentümer ${ }^{(31)}$ des Doms, die Domgemeinde und die Evangelische Kirche der Union (EKU) ${ }^{(32)}$, nicht besonders wünschten. Diesen Umstand nutzte offenbar

25 Bund der Evangelischen Kirchen in der DDR, 15 Jahre Sonderbauprogramm, 95 S. (hier S. 3).

26 Jörg KIrchner, „Das Sonderbauprogramm in der DDR und die ,Kirche im Sozialismus‘. Das Gemeindezentrum in Stralsund Knieper West, 1975-77“, in: Kulturerbe in Mecklenburg und Vorpommern, 4 (2008), Schwerin, 2009, S. 23-34.

27 Zu finden sind oft irreführende Aussagen, dass erst nach 1978, nach dem Staat-Kirche-Gespräch vom 6. März das Sonderbauprogramm in Gang gekommen und Neubauten genehmigt worden seien, während bisher nur Wiederaufbau oder Instandsetzung von Kirchen erfolgten: das ist also falsch.

28 Bund der Evangelischen Kirchen in der DDR, Sonderbauprogramm. Zwischenbericht, im Mai 1980, S. 3.

29 Siehe dazu neuerdings Matthias JudT, Der Bereich Kommerzielle Koordinierung. Das DDR-Wirtschaftsimperium des Alexander Schalck-Golodkowski - Mythos und Realität, Berlin, Christoph Links, 2013.

30 Ludwig GeißEL, Unterhändler der Menschlichkeit, Stuttgart, 1991, zitiert von Christian DüRRAST, Der Evangelische Kirchenbau in der DDR 1969-1989, Magisterarbeit (Nov. 2009) Fach Neuere und Neueste Geschichte am Institut für Geschichtswissenschaften der philosophischen Fakultät I Humboldt Universität Berlin Betreuer: Prof. Dr. Gerd Dietrich, S. 8. Dank an Chr. Dürrast für die Zur-Verfügung-Stellung seines Typoskripts.

31 Die kirchliche Eigentumsfrage ist in Deutschland und Frankreich auf diametral entgegengesetzte Weise geregelt: im Unterschied zu Frankreich, wo die katholischen Kirchen und Gebäude, die vor 1905 gebaut worden sind, der öffentlichen Hand (also der Stadtgemeinde, dem Departement oder dem Staat) gehören, sind die kirchlichen Gebäude in Deutschland Eigentum der Kirchen. In der SBZ/DDR wurde an diesem Status nicht gerüttelt. Im Zuge der Bodenreform 1945 (und danach) wurden die Kirchen dort nie enteignet.

32 Die Evangelische Kirche der Union (EKU) bekam ihren Namen 1954 aufgrund der Umbenennung der Evangelischen Kirche der altpreußischen Union, die 1817 auf Initiative vom preußischen König Friedrich Wilhelm III. aus der Vereinigung der lutherischen und reformierten Gemeinden in Preußen hervorgegangen war. 
Manfred Stolpe zu einem sogenannten Junktim und machte beide Projekte voneinander abhängig ${ }^{(33)}$.

Zurück zur Finanzierungsfrage: das Sonderbauprogramm ist als Teil des Kirchengeschäfts A (sogenanntes Valuta-Programm) zu betrachten. Die BRD organisierte Warenlieferungen (in jährlicher Höhe von 40 Millionen DM), deren Gegenwert den Kirchen in Mark der DDR zur Verfügung gestellt wurde, während das Kirchengeschäft B den Freikauf von Häftlingen oder die Zusammenführung von Familien betraf. „Die Baukosten der Vorhaben wurden in Valuta-Mark abgerechnet. Dabei handelte es sich um die in der DDR übliche statistische Recheneinheit zur Erfassung außenwirtschaftlicher Aktivitäten. Als solche wurde auch die Finanzierung aus dem Westen Deutschlands deklariert“(34).

Das Bauprogramm war vor diesem Hintergrund in die Planungswirtschaft der DDR (Zuteilung von Baukapazitäten, Bilanzierungen, usw.) eingebunden; es mobilisierte staatliche wie kirchliche Bauleitungen, kirchliche wie staatliche Architekten (u.a. aus der Denkmalpflege, zumindest wenn es um die Renovierung von Gebäuden ging, die unter Denkmalschutz standen), staatliche Unternehmen und insbesondere die Außenhandelsfirma Limex Bau. Die Beschaffung von Materialien, die in der DDR schwer zu finden waren, konnte aber auch darüber hinaus direkt über das Diakonische Werk oder Partnergemeinden laufen ${ }^{(35)}$.

Zog aber die SED über die Devisenbeschaffung hinaus weitere Vorteile aus diesem Sonderbauprogramm? Einer SED-Akte aus dem Jahre 1976 ist zu entnehmen, dass die SED sich durch Kirchenneubauten bzw. Gemeindezentren in den Neubaugebieten eine bessere Kontrolle des Gemeindelebens erhoffte. Sie hatte Angst vor den Hauskreisen und hielt diese Form des kirchlichen Lebens für „undurchsichtiger und weniger

33 „Die Evangelischen Kirchen in der DDR und der Wiederaufbau des Doms“, Vortrag des Ministerpräsidenten Manfred Stolpe beim 3. Dom-Kolloquium in Berlin am 4. Februar 2000. http://www.stk.brandenburg.de/reden/2000/0204.html (abgerufen am 5.1.2011).

„Der vom Staat gewünschte Dombau musste als Pfand in Kirchenhand genutzt werden: Es galt, das Dombauprojekt innerhalb des Sonderbauprogramms so zu verankern, dass es von den übrigen Bauvorhaben unterscheidbar blieb, diese nicht infrage stellte, sondern ein zusätzlicher Impuls für deren Durchsetzung blieb.“ S. 5 Webseite; S. 7 „die ,Lokomotive“ Dombau“ ermöglichte dann die Zustimmung zu weiteren Berliner Projekten: dem Dietrich Bonhoeffer Tagungshaus und einem Ergänzungsbau zum KöniginElisabeth-Hospital. Ersteres ist als Tagungshaus der Herrnhuter Brüdergemeine, der pietistisch geprägten Glaubensbewegung der Brüder-Unität in Berlin-Mitte entstanden und später dafür bekannt geworden, dass es die ersten drei Sitzungen des Zentralen Runden Tisches in Berlin am 7., 18. und 22. Dezember 1989 beherbergte. Inzwischen ist es ein Hotel geworden, das an den Verband christlicher Hoteliers e.V. angeschlossen ist.

Letzteres war laut Webseite der aktuellen Einrichtung ein evangelisches „Krankenhaus in Trägerschaft des Diakonischen Werkes mit den Schwerpunkten Innere Medizin und Chirurgie, das im Jahre 1843 gegründet worden war" (http://www.keh-berlin.de/de/geschichte abgerufen am 8.7.2013).

34 J. Kirchner, „Das Sonderbauprogramm in der DDR“ (Anm. 26), S. 25. Er fährt fort: „Der jeweilige Umrechnungskurs der Valuta-Mark blieb unveröffentlicht, so dass die jeweiligen Kostenangaben nur eingeschränkte Aussagekraft besitzen und eher als Vergleichsgrößen von Interesse sind.“

35 Verschiedene Akten aus dem Evangelischen Landeskirchlichen Archiv Berlin (ELAB) über die Friedensgemeinde Eisenhüttenstadt geben einen guten Einblick in diese Problematik und illustrieren sie mit ganz konkreten Beispielen. Siehe Akten unter der Signatur K1257 und I31, Va. Bd. VIII 1982-1984. 
überschaubar als in offiziellen Kirchenräumen“ (36). Die Hauskreise waren nämlich Gesprächskreise (Bibelkreise o.ä.), die sich in regelmäßigen Abständen bei den Teilnehmern selbst abwechselnd trafen. Eine Unterwanderung der sozusagen dezentralen Hauskreise kam der SED schwieriger vor als bei zentralen Veranstaltungen etwa im Gemeindehaus.

In derselben Akte ist übrigens auch zu erfahren, dass die Genehmigung des Gemeindezentrums für Eisenhüttenstadt 1976 reibungslos erfolgte, wohl weil es keine schwierige, widerspenstige Gemeinde war.

Darüber hinaus waren Sonderbauprogramm und Kirchenbau überhaupt für die SED ein Mittel, symbolisches Kapital zu schlagen. Ihre pragmatischere Kirchenpolitik und das damit verbundene Sonderbauprogramm sind also auch in den Zusammenhang mit dem Ringen der DDR nach internationaler Anerkennung im Zuge der Entspannungspolitik und der Unterzeichnung der deutsch-deutschen Verträge einzuordnen. Die Entwicklung eines eigenen Nationalbewusstseins, des Bewusstseins einer sozialistischen deutschen Nation, ging auch damit einher. In diesem Rahmen wurde die Rezeption vorhandener Kulturdenkmäler - unter anderem aus dem Raum der Kirche - im Zeichen des dialektischen Begriffspaars „Erbe/Tradition“ intensiviert. Es kam der Denkmalpflege zugute, die selbst von längerfristigen Entwicklungen profitierte: das internationale Jahr des Denkmals 1975, in dem das Denkmalschutzgesetz der DDR verabschiedet wurde, wird allenthalben ${ }^{(37)}$ als eine wichtige Zäsur in diesem Bereich betrachtet.

Dass die sonst übliche Periodisierung des Staat-Kirche-Verhältnisses in Bezug auf Kirchenbau nicht immer greift, oder dass die sich aus dem Eisenhüttenstädter Fall ergebenden Erkenntnisse nicht auf die ganze DDR übertragbar sind, möchte ich hier noch kurz andeuten. 1957 veröffentlichte der Architekt und Kirchenoberbaurat im Kirchlichen Bauamt Potsdam, Winfried Wendland, eine kleine reich illustrierte Abhandlung über den „Kirchenbau in dieser Zeit“, aus der hervorgeht, dass eine Vielzahl von Kirchen in Dörfern, in Städten bis 1954 und 1956 oder sogar 1957 wiederaufgebaut oder neugebaut wurden. Fest steht, dass eine Reihe von Wiederaufbaumaßnahmen noch in die Zeit der den Kirchen eher wohlgesonnenen Sowjetischen Militäradministration fallen ${ }^{(38)}$. Fest steht auch, dass die SED damals im Juni 1946 im Hinblick auf Wahlen auch noch Anweisungen gab zur Unterstützung von „Gesuche(n)“ zur „Renovierung von beschädigten Kirchengebäuden oder zur Anschaffung von Gegenständen, die für den Gottesdienst notwendig sind“(39).

36 Siehe Dok. 44 „Information der Arbeitsgruppe Kirchenfragen im ZK der SED für Paul Verner und Hans Seigewasser vom 1.6.1976 über Bestrebungen der Kirche zur Abgabe von Kirchengebäuden sowie zur Errichtung kirchlicher Gemeindezentren in Neubaugebieten der DDR“, in: F. HARTwEg (Hg.), SED und Kirche (Anm. 22), S. 280-284 (Zitat S. 282).

37 Siehe z.B. Gerhart PASCH, „Erhaltung und Restaurierung von Kirchen in der DDR“, in: Herbergen der Christenheit: Jahrbuch für deutsche Kirchengeschichte, Leipzig, Evangelische Verlagsanstalt, 1996, S. 192208 (besd. S. 204); Rudolf MAU, Der Protestantismus im Osten Deutschlands 1945-1990, Leipzig, evangelische Verlagsanstalt, 2005, S. 122.

38 Dürrast erwähnt sogar den Bau von neun Kirchen im Rahmen des ,ersten von Otto Barning entworfenen Notkirchenprogramms“. Ch. DürRast, Der Evangelische Kirchenbau (Anm. 30), S. 15.

39 M. Tillmann, Das Verhältnis von Staat und Kirche (Anm. 9), S. 12, zitiert Gerhard Besier, Der SED-Staat und die Kirche. Der Weg in die Anpassung, München, Bertelsmann, 1993, S. 30. 
Fest steht außerdem, dass Otto Nuschke bis zu seinem Tod 1957 eine diesbezügliche Vermittlungsrolle spielen konnte. Auf ihn ist der sogenannte Nuschke-Fonds zurückzuführen, der jährlich 1,2 Millionen Mark Zuschüsse für Baukosten verteilen konnte. Dieser Fonds, der ab 1960 vom Ministerium für Kultur verwaltet wurde, blieb bis zum Ende der DDR erhalten. Die Situationen müssten also im Einzelfall geprüft werden. Die politischen Rahmenbedingungen waren auf dem Land, in der Kleinstadt, in der Großstadt unterschiedlich. Eine Reihe von Kirchen sind wohl nur aufgrund des freiwilligen Einsatzes der Gemeindeglieder und der mehr oder weniger improvisierten Hilfe vom Westen wieder aufgebaut worden ${ }^{(40)}$.

Es besteht also die Vermutung, dass die Geschichte des Kirchenbaus nach einem ihr eigenen Rhythmus und unter etwas anderen Vorzeichen verlief als andere Bereiche des kirchlichen Lebens oder des Staat-Kirche-Verhältnisses. Eine Studie vom Staatssekretariat für Kirchenfragen 1960 lässt zumindest diese Annahme zu: nach dieser Studie ${ }^{(41)}$ seien von 9824 evangelischen Kirchen, worunter 2678 schwer beschädigt und 200 total zerstört waren, 1400 der beschädigten Kirchen bis zum Jahre 1957 mit staatlicher Hilfe in Stand gesetzt bzw. neuaufgebaut und 52 neu erstellt worden. Die Gegenbeispiele später Sprengung von schon wiederaufgebauten Kirchen, wie zum Beispiel der Universitätskirche in Leipzig $1968^{(42)}$, der Christuskirche in Rostock $1971^{(43)}$ oder gar der JakobusKirche in Dessau $1977^{(44)}$ weisen auch ex negativo auf diesen asynchronen Rhythmus hin. Eine Geschichte des Kirchenbaus in der DDR bleibt noch zu schreiben ${ }^{(45)}$ !

\section{Schlusswort}

Kommen wir auf die Eingangsfrage zurück. Was lehrt uns das Beispiel Eisenhüttenstadt in Bezug auf das mögliche gegenkulturelle Potential der Religion in der DDR? Wir können hier zweierlei festhalten. Der Fall Eisenhüttenstadt hat exemplarisch gezeigt, inwiefern bauliche Probleme in der DDR als politische Instrumente gehandhabt

40 Ch. Dürrast, Der Evangelische Kirchenbau (Anm. 30), S. 23-25. Er erwähnt z.B. die sogenannten GustavAdolf-Kirchen, die vom Gustav-Adolf-Werk mitfinanziert wurden.

41 M. Tillmann, Das Verhältnis von Staat und Kirche (Anm. 9), S. 12, zitiert eine Studie vom Staatssekretariat für Kirchenfragen 1960, „Staat und Kirche in DDR. Eine Dokumentation“, 36 S. (im Brandenburgischen Landeshauptarchiv unter folgender Signatur zu finden: Bez. Ffo. Rep. 601, Sign. 8221). Eine andere Studie wird von Dürrast zitiert, aber ohne Datum (Der Evangelische Kirchenbau [Anm. 30], S. 21).

Katrin LöFfler, Die Zerstörung: Dokumente und Erinnerungen zum Fall der Universitätskirche Leipzig, Leipzig, Benno-Verl., 1993, 234 S.; Ehrhart NeuberT, Geschichte der Opposition in der DDR 1949-1989, Berlin, Links, 1997, S. 177-181: der Plan eines Abrisses reichte bis Ende der 1950er / Anfang der 1960er Jahre zurück.

43 Georg M. Diederich, Aus den Augen, aus dem Sinn. Die Zerstörung der Rostocker Christuskirche 1971, Rostock, Ed. Temmen, 1997, 240 S.

44 Ch. Dürrast, Der Evangelische Kirchenbau (Anm. 30), S. 15.

45 Erste wichtige Ergebnisse auf diesem Weg sind Christian Dürrast (Der Evangelische Kirchenbau, ebd.) und Henriette von Preuschen zu verdanken: Henriette von Preuschen, Der Griff nach den Kirchen: Ideologischer und denkmalpflegerischer Umgang mit kriegszerstörten Kirchenbauten in der DDR, Worms, Wernersche Verlagsgesellschaft, 2011, 258 S. (zugl.: Cottbus, Techn. Univ. Diss., 2010); Henriette von Preuschen, „Zum ideologischen und denkmalpflegerischen Umgang mit kriegszerstörten Kirchen in der DDR: Die Bezirkshauptstadt Magdeburg“, in: Sigrid Hofer (Hg.), Grenzgänge zwischen Ost und West, Dresden, Sandstein Verlag, 2012, S. 108-126. 
wurden $^{(46)}$ und wie brisant die Kirchenbaufrage in der DDR gewesen ist. Über die Neubauten hinaus könnte man diese Aussage genauso gut auf die Denkmalpflege (von Kirchen) beziehen sowie auf andere Bereiche des christlichen kulturellen Erbes. Es ließen sich hier zahlreiche weitere Beispiele anführen.

„Wer baut, ist noch nicht gestorben. [...] Gemeinden wissen auch, da $\beta$ wertvolle Tradition gegenüber den Außenstehenden eine besondere Anziehung ausübt“(47).

Indem dieses Zitat von Manfred Stolpe die Baufrage mit derjenigen der kulturellen Ausstrahlung verknüpft und damit indirekt die Rolle der Kirche als wichtiger Kulturträgerin in der DDR betont, faßt es vorzüglich zwei Seiten einer Medaille und ein Kernargument unserer Untersuchung zusammen: Kirche wurde zwar durch Ideologie und Politik des Parteistaates in eine gegenkulturelle Rolle eingezwängt. Die Aura, die ihr aber somit als kulturellem Gegenpol zuwuchs, erfolgte quasi ohne ihr Zutun durch Pflege ihrer Tradition und Kultur.

Ausgehend vom hier herangezogenen wörtlichen Sinne des Begriffs Gegenkultur sind im Rückblick das Hin und Her, die Austauschprozesse oder Umkehrbewegungen zwischen offizieller Leitkultur und Randkultur im Osten Deutschlands besonders auffällig: während die christliche Religion und Kultur als frühere Leitkultur in der DDR bald zum Feind erklärt und an den Rand gedrängt wurde, kehrten sich die politisch-gesellschaftlichen Symbole nach der politischen Wende 1989 um: so wurde unter völlig veränderten Vorzeichen der ehemalige und langjährige Pfarrer der evangelischen Gemeinde Eisenhüttenstadt in den Kreis der ersten Honoratioren der Stadt erhoben. Er wurde zum ersten Ehrenbürger der Stadt, dem der erste Eintrag ins Goldene Buch der Stadt galt. 2006 wurde schon zu Lebzeiten eine Straße nach ihm genannt.

\section{Zusammenfassung}

Der Beitrag untersucht anhand des Fallbeispiels von einem evangelischen Kirchenbau in Eisenhüttenstadt, inwiefern der gegenkulturelle Ansatz in Bezug auf Religion und Kirchen in der DDR relevant ist. In dieser sozialistischen Vorzeigestadt des DDR-Regimes war der Bann über die Kirchen besonders ausgeprägt und das kirchliche Leben unerwünscht. Dass Religion und Kirchen vom DDR-Regime als Gegenkultur im wörtlichen Sinne betrachtet wurden, lässt sich dort an der wechselvollen Entstehungsgeschichte eines „Massivkirchenbaus" erkennen, der das 30jährige Provisorium (Zelt, Wohnwagen, Kirchbaracke aus Holz) ersetzen sollte. Dass das neue Gemeindezentrum 1981 schließlich eingeweiht werden konnte, lag an der Auflockerung der Beziehungen zwischen Staat und Kirche seit den 1970er Jahren und konkret am Kirchensonderbauprogramm, das zwischen Ost und West ausgehandelt wurde. Die Stunde der Pragmatiker hatte nun geschlagen. Diese Entwicklung zeigt aber auch, dass die besondere Ausstrahlung der Kirche als Kulturträgerin in der DDR intakt geblieben war.

46 Siehe auch allgemein G. PAsCH, „Erhaltung und Restaurierung von Kirchen“ (Anm. 37), S. 193.

47 Zwischenbericht 1976 (Anm. 24), S. 2. 


\section{Résumé}

Partant de l'histoire mouvementée du projet de construction d'un temple protestant à Eisenhüttenstadt, l'article étudie la pertinence du concept de contre-culture rapporté au rôle de la religion et des Églises en RDA. Dans cette ville sidérurgique, vitrine idéologique du régime communiste, la volonté d'ostraciser les Églises s'est fait clairement sentir. En témoignent les vicissitudes qu'a connues le projet de temple "en dur » destiné à remplacer les solutions provisoires ayant eu cours pendant 30 ans (tente, caravane, "temple-baraque » en bois). La construction d'un centre paroissial, inauguré en 1981, est finalement due à l'assouplissement des relations Église/État depuis les années 1970 et à la négociation entre l'Est et l'Ouest d'un programme spécial de construction d'églises. L'heure des pragmatistes avait sonné. Mais cette évolution montre aussi combien le rayonnement des Églises en tant que vecteur de culture était intact en RDA. 